\title{
Optimal Scheduling of Doctors Outpatient Departments Based on Patients' Behavior
}

\author{
Zongwei Ren, Haini Guo, Shizhen Bai, and Peng Li \\ School of Management, Harbin University of Commerce, No. 1, Xuehai Street, Harbin 150028, China \\ Correspondence should be addressed to Zongwei Ren; rzw79@163.com
}

Received 16 June 2016; Revised 11 September 2016; Accepted 21 September 2016

Academic Editor: Junhu Ruan

Copyright (C) 2016 Zongwei Ren et al. This is an open access article distributed under the Creative Commons Attribution License, which permits unrestricted use, distribution, and reproduction in any medium, provided the original work is properly cited.

\begin{abstract}
The low operational efficiency in the field of medical and health care has become a serious problem in China; the long time that the patients have to wait for is the main phenomenon of difficult medical service. Medical industry is service-oriented and its main purpose is to make profits, so the benefits and competitiveness of a hospital depend on patient satisfaction. This paper makes a survey on a large hospital in Harbin of China and collects relevant data and then uses the prospect theory to analyze patients' and doctors' behavioral characteristics and the model of patient satisfaction is established based on fuzzy theory with a triplet $\alpha / \beta / \gamma$. The optimal scheduling of clinic is described as a problem with the rule of first come, first served which maximizes patient satisfaction for the main goal and minimizes operating costs for the secondary goal. And the corresponding mathematical model is established. Finally, a solution method named plant growth simulation algorithm (PGSA) is presented. Then, by means of calculating of the example and comparing with genetic algorithm, the results show that the optimum can be reached; meanwhile the efficiency of the presented algorithm is better than the genetic algorithm.
\end{abstract}

\section{Introduction}

With the improvement of living standards, people tend to pursue a higher quality of life, and the limitation of treatment level in secondary hospitals and community hospitals leads to a serious problem of registered queue in high-level hospitals. There are usually three types of registration in hospitals, namely, general-clinic, specialist-clinic, and emergencyclinic. How to allocate the number of doctor outpatient departments for every type of registration is very essential for operational efficiency in hospital and has a close relationship with patient satisfaction at the same time. Because of the different-type registrations, this paper makes optimal scheduling for the number of doctor outpatient departments considering the patient behavioral characteristics in order to minimize patient waiting time and increase their satisfaction and reduce operating costs.

The main goal of current researches in hospital is lowering costs and running better. Many papers achieved these goals from the perspective of the effective utilization of resource. The resource can be divided into human resource and material resource. Researchers used different methods to optimize the material resource such as Operating Room (OR), multihospital network, and limited number of wards [1-4]. All of the methods mentioned above made some assumptions which can simplify the solution of the problems although there were some discrepancies with reality. Vile et al. [5] investigated several interrelated advanced statistical and operational research methods and sought to predict future demands better, recommended minimum staffing requirements, and generated low-cost rosters. This article aims at optimizing the human resource; however, it only considered a single class of customers and its methods were only reliable under limited conditions. Wright and Mahar [6] studied the staff nurse scheduling in order to improve nurse satisfaction. It was also about the use of human resource and there were two limitations in this paper. First, it did not contain other scheduling policies and another limitation was that they only centralized scheduling decisions across three units in their study.

In many literatures, patients' waiting time is a major factor to be considered because it is closely linked with the quality of hospital operation. Now the hospital aims to deliver the highest quality of care, so reducing waiting time 
of patient is important [7-9]. These papers gave a function to compute waiting time or gave a new concept called "breakin-moments" (BIMs) to reduce waiting time. However the latter one is only considering the emergency surgery. In the case of BIM, further researches are also needed to investigate whether inserting breaks between surgeries which can increase the stability of the schedule is needed. From another viewpoint, Shuangxi et al. [10] analyzed historical data of patients' medical treatment time in the last three years, concluded its regularity, and then calculated the number of doctors' configuration. But the research focused on children's regularity based on large amounts of data of the children's hospital which are much different from other high-level hospitals.

Queuing theory is always being used to deal with the problem of minimum waiting time. Lakshmi and Iyer [11] reviewed the contributions and applications of queuing theory in the field of health care management problems and proposed a system of classification of health care areas which are examined with the assistance of queuing models. The research's goal is to provide sufficient information to analysts who are interested in using queuing theory to model a health care process and who want to locate the details of relevant models.

Our research applies prospect theory and membership functions of patient satisfaction to build a model, taking into account the patient behavioral characteristics. Xuping et al. [12] made optimal scheduling of emergency supplies considering the public psychological perception of risk and Yang et al. [13] proposed interference management models of single machine scheduling from multiple angles actors. None of these articles used prospect theory into medicinal service.

It is worth noting that the optimization of service industries is different from manufacturing industries; the former one is always accompanied by the participation of people, and usually it will be affected by people's behavior and subjective emotion, so the hospital should focus on patient satisfaction. Combined with prospect theory and fuzzy theory and taking into account the patient behavioral characteristics at the same time, we have the research of the optimal scheduling for doctor outpatient departments in one high-level hospital of Harbin. And the starting point matches the urgent social medical problems in China.

The remainder of this paper is organized as follows. In Section 2, we introduce the queue classification analysis based on satisfaction degree of patients; Section 3 focuses on the description of problem and establishment of model; a genetic algorithm is described in Section 4; a medical case whose data is collected in a Harbin hospital is given in Section 5, together with simulation results. Conclusions are drawn in Section 6.

\section{Queue Classification Analysis Based on Patient Satisfaction Degree}

2.1. The Hospital Registration Type Analysis. The types of hospital registration always include general-clinic, specialistclinic, and emergency-clinic. Under normal circumstances, the fee of these three types of registrations increases in turn.
In general, the number of emergency-clinics is much less than the former two. The difference between general-clinic and specialist-clinic is that doctors' experience and technology are relatively richer in specialist-clinics and the registration's fee is higher. Usually, if the patient's situation is not too serious, he will choose general-clinic; if the patient needs comprehensive thorough check, he will choose specialistclinic; if there is urgent situation, the patient will choose emergency-clinic. Patients can select the appropriate type of registrations according to their own conditions and actual demands. Due to the needs of doctor outpatient departments are different in each type of registration; this paper makes optimal scheduling under actual situations. The following is description of registration categories:

(1) general-clinic A: suitable for the milder clinical symptoms of patients;

(2) specialist-clinic B: suitable for the heavy condition and needs a thorough examination of patients;

(3) emergency-clinic C: suitable for the emergency condition of patients.

The emphasis of this article is on the lean staffing of doctor outpatient departments according to different needs of patients under the varied registration. That is, how much doctors should be arranged for each type of registrations in order to minimize patient waiting time and increase their satisfaction and reduce operating costs.

2.2. Patient Behavior Analysis. In general, different patients in a hospital have different expected waiting time when they are queuing and here are several factors affecting it. First, people with different personality have different expectation of waiting time. Character is the factor that influences patients' emotion themselves. The expected waiting time of patients who have urgent character is much shorter than the patients who have slow character. Second, it depends on patients' different conditions. Usually, the expected waiting time of patients who have serious illness is much shorter than the patients whose illness is lighter. But it does not adapt to all patients absolutely and the length of expected waiting time is different from person to person. If patients' actual waiting time exceeds the length of expected waiting time, the patients will produce bad emotion such as anxiety and discontent. So we divide patient satisfaction degree into three levels: satisfaction (the value is equal to 1 ), dissatisfaction, and very dissatisfaction (the value is equal to 0 ). We make survey of the patients in a high-level hospital in Harbin and collect data through the way of interview and questionnaire. And then arrange the data to draw the figure of patient waiting time distribution in the same satisfaction degree, as shown in Figures 1-3.

About patients' satisfaction level, the following points should be noted.

(1) The hospital is a special service agency; its service system is different from dining hall or bank and its process is more complicated. Patients can choose any type of registration for treatment, so after receiving 


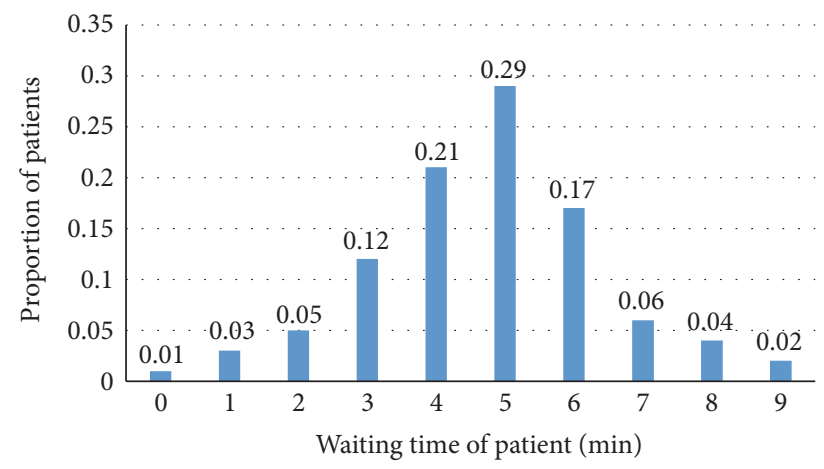

FIGURE 1: The distribution of waiting time under satisfaction level.

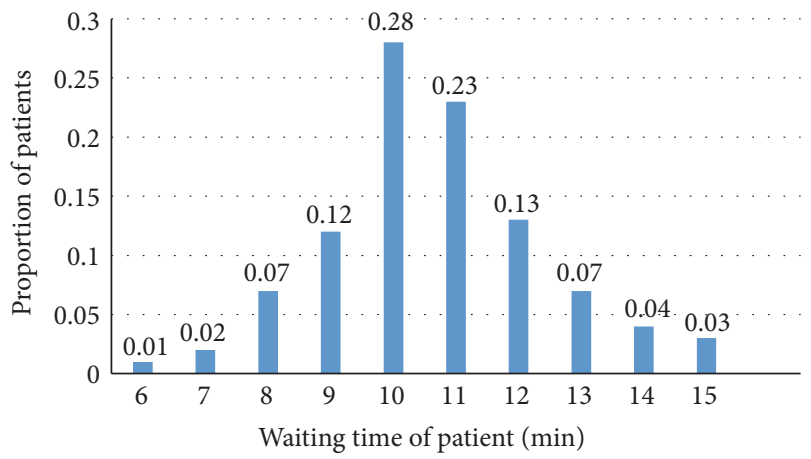

FIGURE 2: The distribution of waiting time under dissatisfaction level.

the first service from one doctor outpatient department, the patients are likely to go to other service systems such as pharmacy and radiology and, then through the queuing, wait to receive service again and finally they may leave or go back to the first queuing system. In this paper, the satisfaction level of patients we study only refers to the fact that patients produce negative emotions when they get into queuing system for the first time.

(2) In the whole process of treatment, patients not only have to queue for many times, but also through a number of staff positions in hospital. So the factors that influence patient satisfaction degree not only contain the length of waiting time at the first time entering into queuing system, but also will be affected by other aspects of service quality. This paper highlights the number of doctor outpatient departments for every type of registration ignoring other factors and only considers the influence that the length of waiting time brings to patient satisfaction for the first time of queuing.

2.3. Doctor Behavior Analysis. If the number of doctor outpatient departments has irrational allocation for every type of registration, it will not only make patients wait long time, but also affect the doctor work's efficiency and accuracy. On one hand, if more doctor outpatient departments are opened,

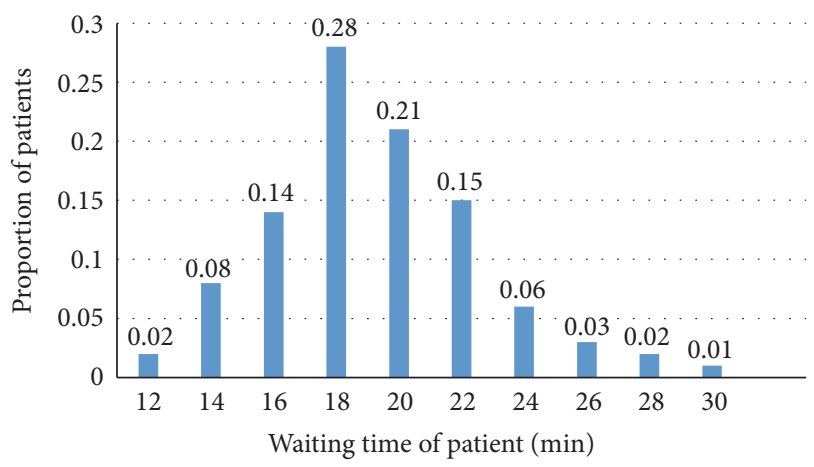

FIGURE 3: The distribution of waiting time under very dissatisfaction level.

it will greatly shorten the waiting time of patients, but it increases operating costs of the hospital and causes the waste of doctor resources. On the other hand, if setting up the low cost as the center goal of hospital and reducing the number of doctor outpatient departments, it will lead to serious queuing phenomenon of patients and increase the doctors' workload. As a result, it will let the misdiagnosis rate rise because of no time to rest for doctors and ultimately affect patient satisfaction. What is more, it can make the relationship between doctor and patient become more nervous.

2.4. Patient Satisfaction Model. The major research of customer satisfaction is the relationship between their psychological characteristics and activities; thus human behavior characteristics should be mainly considered to improve the customer satisfaction. There are many valuable researches about human behavior. Zhihong et al. [14] used the similarity of Tanimoto to measure customer behavior and then designed genetic clustering algorithm to divide customer groups and studied different purchasing behaviors of these groups. They designed a feature extraction method of multigroup based on genetic algorithm. We can apply this method into many fields of customers. Chun and Yiping [15] proposed an agent-based modeling and simulation method to analyze customer behavior and the effectiveness of personalized recommendation strategy. This paper only considered the mobile electronic commerce situation. Tingting et al. [16] made a survey of a large supermarket and analyzed customer behavior characteristics based on prospect theory and then built corresponding mathematical model to make optimal scheduling for retail checkout. The situation of this research was similar to hospital. All these articles are involved in customer satisfaction.

The environment of hospitals, staff service attitude, and the length of waiting time can affect patient satisfaction. This paper analyzes patient and doctor behavior characteristics by using the theory of behavior science and then establishes patient satisfaction function of waiting time.

People's perception of behavior and satisfaction is fuzzy and uncertain. The prospect theory has great advantage to describe people behavior characteristics; we can accurately 


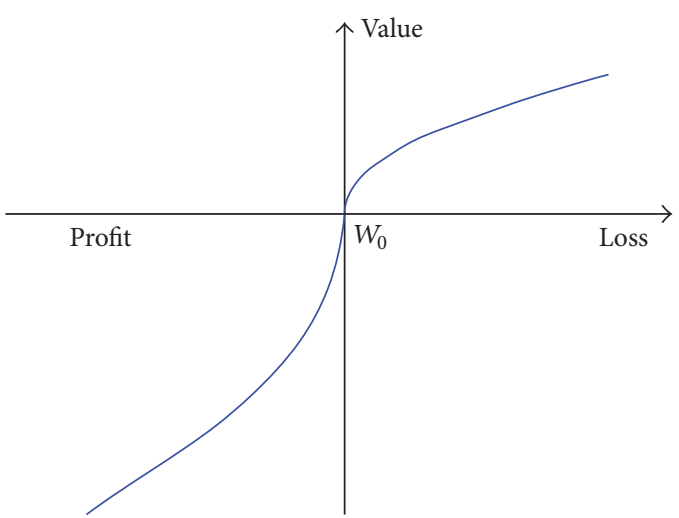

FIGURE 4: The value function diagram.

build descriptive model through analyzing patient satisfaction degree based on this theory. However, the prospect theory lacks mathematical theory to support. Fuzzy theory has good mathematical theory basis, but it cannot accurately describe patient behavior characteristics. So we combined the two methods effectively to establish patient satisfaction function.

Prospect theory combines psychology with economics effectively and uses the value function to represent policymakers' subjective value. Its core is the selection of reference point. The evaluation of effectiveness about prospect theory is based on the reference point $W_{0}$, and when the result of event is $W$, we can use amplitude and direction which $W$ deviates $W_{0}$ to evaluate effectiveness. The formula is $W-W_{0}$. We treat the expected waiting time of patient as $W_{0}$ and according to the issue we study, we can make the plot describe the problem as shown in Figure 4.

When patient's actual waiting time is longer than expected waiting time (reference point $W_{0}$ ), there will be a loss; otherwise it represents profit.

The value function transports the surface value into decision value. This paper treats the expected waiting time of patient as a reference point because patients pay more attention to the value of difference between expected waiting time and actual waiting time rather than the result itself. According to the prospect theory which Kahneman and Tversky [17] have put forward, we establish the value function of patient $j$ ( $j$ means any one of patients):

$$
V_{j}(x)=\left\{\begin{array}{ll}
x^{\alpha}, & x \geq 0, \\
-\lambda(-x)^{\beta}, & x<0,
\end{array} \quad j=1,2, \ldots, n,\right.
$$

where $\alpha, \beta$ are coefficients of attitude to risk. The bigger $\alpha$ and $\beta$ are, the more likely decision makers are to pursue risk. $\lambda$ is the loss aversion coefficient and when $\lambda>1$, it means that the decision makers are more sensitive to loss. Kahneman and Tversky's study concluded that $\alpha=\beta=0.88$ and $\lambda=2.25$ which are consistent with empirical data. There are also other researchers showing that $\beta$ is bigger than $\alpha$. Jianmin [18] proposed that $\alpha=1.21, \beta=1.02$, and $\lambda=2.25$ under the situation of China and these results based on China actual conditions are different from foreign countries, so they are more valuable.

As the patient's perception of events is fuzzy and uncertain and the prospect theory can only describe this uncertainty qualitatively, it cannot make decision accurately. So this paper uses the membership function of fuzzy theory to obfuscate objective function. The specific steps are as follows.

Step 1. Determine the set of evaluations $T=\left(T_{1}, T_{2}, T_{3}\right)=$ $\left(1, T\left(t_{j}\right), 0\right) . T$ represents the satisfaction degree set; each value in this set means satisfaction, dissatisfaction, and very dissatisfaction. $t_{j}$ means the actual time that patient $j$ leaves this queuing system; $d_{j}$ means the expected time that patient $j$ leaves this queuing system; and $M_{j}=t_{j}-d_{j}$ means the lag time of the patient $j$. When $t_{j}>d_{j}$, it means a loss; otherwise there will be profit.

Step 2. Determine the threshold of each satisfaction degree. According to the analysis of survey results, the threshold of satisfaction degree is related to the expected waiting time $\left(d_{j}\right)$ of patient $j$, coefficient of attitude to risk $(\beta)$, and loss aversion coefficient $(\lambda)$. There is a formula $R_{j}=d_{j}+(1 / \lambda)^{1 / \beta}$ describing the relationship between them which is concluded by other researchers. When $t_{j} \geq R_{j}$, the value of satisfaction membership degree equals $0\left(T\left(t_{j}\right)=0\right)$. When $0 \leq t_{j}<d_{j}$, the value of satisfaction membership degree equals $1\left(T\left(t_{j}\right)=\right.$ $1)$. When $d_{j} \leq t_{j}<R_{j}$, the people prefer to pursue risk when there is a loss. According to prospect theory, we can make derivation under formula (1):

$$
\begin{aligned}
T\left(t_{j}\right) & =1-\left(-V_{j}(x)\right)=1+V\left(-t_{j}+d_{j}\right) \\
& =1+\left[-\lambda\left(-\left(-t_{j}+d_{j}\right)\right)^{\beta}\right]=1-\lambda\left(t_{j}-d_{j}\right)^{\beta} .
\end{aligned}
$$

This paper uses the typical parameters $\alpha=1.21, \beta=1.02$, and $\lambda=2.25$.

Step 3. Establish satisfaction function.

In the end, the satisfaction membership function can be established, as in the following formula. Figure 5 can clearly describe trend of the function:

$$
T\left(t_{j}\right)= \begin{cases}1, & 0 \leq t_{j}<d_{j} \\ 1-\lambda\left(t_{j}-d_{j}\right)^{\beta}, & d_{j} \leq t_{j}<R_{j}, \\ 0, & t_{j} \geq R_{j} \\ & j=1,2, \ldots, n .\end{cases}
$$

According to formula (1) and formula (3) and $R_{j}=d_{j}+$ $(1 / \lambda)^{1 / \beta}$ which reveals the relationship between threshold $R_{j}$ and expected waiting time $d_{j}$ of patients, we can deduce that $\lambda\left(R_{j}-d_{j}\right)^{\beta}=1$. As the domain of deduction is $d_{j} \leq t_{j}<R_{j}$ and the value of $\lambda$ is equal to 2.25 , the value interval of $\lambda\left(t_{j}-\right.$ $\left.d_{j}\right)^{\beta}$ is $(0,1)$. So it verifies correctness of the above model we build. 


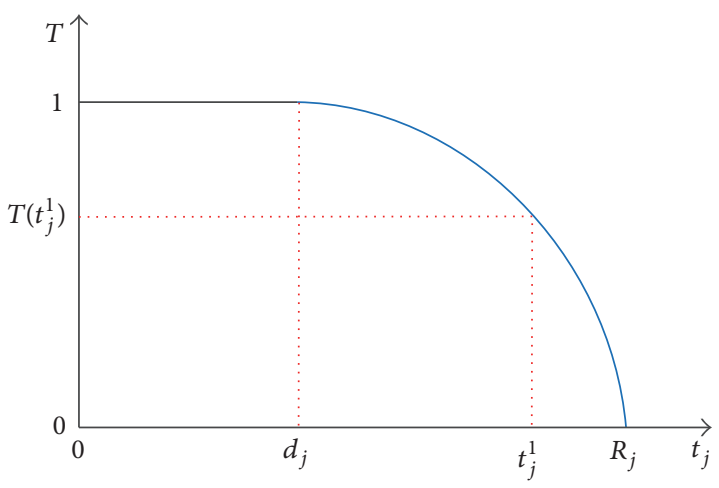

FIGURE 5: The satisfaction membership function diagram of patient $j$.

\section{Description of Problem and Establishment of Model}

3.1. Description of Problem. Hospital managers need to adjust the number of doctor outpatient departments according to patients' number and their registration type in order to improve patient satisfaction and reduce operating costs. Hospital belongs to service-oriented industry, and its highest goal is patient satisfaction. Thus, the problem can be described as maximizing patient satisfaction for the main goal and minimizing operating costs for the secondary goal. So it is a multiobjective scheduling problem. We can use a triple $\alpha / \beta / \gamma$ to describe this scheduling problem. The domain of $\alpha$ mainly describes the environment of resources; the domain of $\beta$ mainly analyzes the constraints; and the domain of $\gamma$ describes optimal scheduling goals.

3.1.1. Environment of Resources ( $\alpha$ Domain). Patients can choose different types of registration according to their own conditions and demands. The service level is different under each type of registration (usually, the doctors of specialistclinic have higher qualification and rich experience). As this paper focuses on solving the optimal scheduling problem of doctor outpatient departments, we make the following description. $n$ indicates the total number of doctor outpatient departments; $a$ indicates the number of doctor outpatient departments under general-clinic; $b$ indicates the number of doctor outpatient departments under specialist-clinic; and $c$ indicates the number of doctor outpatient departments under emergency-clinic. Figure 6 shows the simple process of patient treatment in hospital.

$\mathrm{A}, \mathrm{B}$, and $\mathrm{C}$ respectively represent general-clinic, specialist-clinic, and emergency-clinic.

3.1.2. Constraints and Rules ( $\beta$ Domain). Each patient should choose the appropriate registration type according to their own actual situation to accept service in doctor outpatient departments, so there is constraint of using different registration types. Let $N_{j}$ describe this constraint. Before patient $j$ entering into queuing system, the previous patient $j-1$ has priority to receive service. That means the present patient

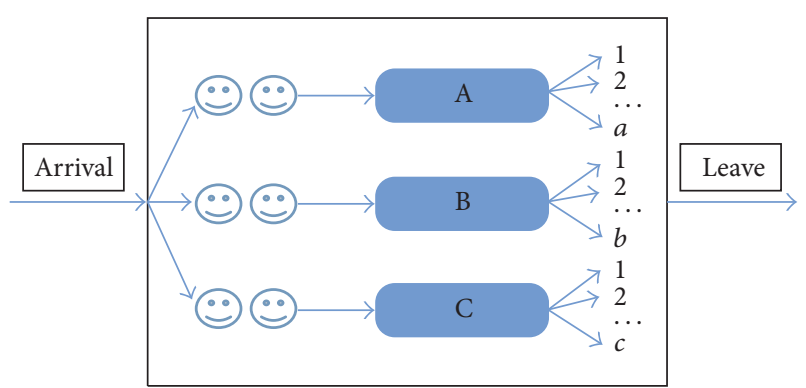

FIGURE 6: The treatment process diagram of patient $j$.

must have finished accepting service before the next patient receives service. In other words, the rule is first come first served (FCFS).

3.1.3. Optimal Scheduling Target ( $\gamma$ Domain). The hospital's ultimate target is to make profit as a service-oriented institution. For hospitals, the goal is to reduce operating costs as far as possible, but it is not a major one. The service quality is directly related to performance of hospital. If patients produce bad emotion because of long waiting time, it will influence the revenue of hospital. So improving patient satisfaction is the most important. Patient satisfaction is affected by many factors, but this article focuses on the waiting time that patient enters into the queuing system at first time, ignoring the interference of other factors. The first goal is to maximize patient satisfaction and we use the formula $\max \sum_{j=1}^{m} T\left(t_{j}\right) / m$ to express. $m$ indicates the total number of patients. The second goal is to minimize the number of doctors, and we use the formula $\min \sum_{j=1}^{m}\left(a_{i j}+b_{i j}+c_{i j}\right)$ to express. $\sum_{j=1}^{m} a_{i j}, \sum_{j=1}^{m} b_{i j}$, and $\sum_{j=1}^{m} c_{i j}$, respectively, represent the number of doctor outpatient departments under each type of registration.

3.2. Parameter Design. $n$ means the total number of doctor outpatient departments; $m$ means the total number of patients; $i$ means any one of the doctor outpatient departments $(i \in[1, n]) ; j$ means any one of patients $(j \in[1, m])$; $\eta$ means the number of patients arrived at the hospital in a unit time and we treat it as arrival rate; $\mu$ means the number of patients finished accepting service in a unit time; it was treated as service rate and it can be regarded as doctor's work efficiency; $c_{i}$ means the doctor's work time of any one doctor outpatient department and every doctor has fixed department; $p_{j}$ means the length of time that patient $j$ received service and it is related to the patients' situation; $r_{j}$ means the submitted time of report for patient $j ; t_{j}$ means the actual time that patient $j$ leaves this queuing system; $d_{j}$ means the expected time that patient $j$ leaves this queuing system; and $M_{j}$ means the lag time of the patient $j$. The decision variables are as follows:

$$
x_{j}= \begin{cases}1, & \text { The patient is being served, } \\ 0, & \text { other cases, }\end{cases}
$$


$D_{i j}= \begin{cases}1, & \text { The type of registration is } \mathrm{A}(i \in \mathrm{A}), \\ 2, & \text { The type of registration is } \mathrm{B}(i \in \mathrm{B}), \\ 3, & \text { The type of registration is } \mathrm{C}(i \in \mathrm{C}) .\end{cases}$

\subsection{Basic Assumptions}

Assumption 1. Each patient must select only one doctor outpatient department to get service.

Assumption 2. Do not consider the case that the patients go back to the queuing system for the second time.

Assumption 3. The patients will leave when waiting time exceeds a certain range.

Assumption 4. Each type of registration follows the principle of FCFS and the patients cannot change the queue when they selected.

3.4. Model Design. Treating doctor outpatient departments scheduling problem as parallel machine scheduling problem with different speed, we establish the following models:

$$
\begin{aligned}
& \left(\frac{Q_{m}}{\operatorname{prec}}, \frac{N_{j}}{\max \sum_{j=1}^{m} T\left(t_{j}\right) / m(\text { opt })},\right. \\
& \left.\min \sum_{j=1}^{m}\left(a_{i j}+b_{i j}+c_{i j}\right)\right) .
\end{aligned}
$$

The corresponding mathematical models are as follows:

$$
\begin{aligned}
& \max \quad Z=\frac{\sum_{j=1}^{m} T\left(t_{j}\right)}{m}, \\
& \min \quad W=\sum_{j=1}^{m}\left(a_{i j}+b_{i j}+c_{i j}\right), \\
& \text { S.t. } \quad M_{j}=\max \left(t_{j}-d_{j}, 0\right), \\
& \quad T\left(t_{j}\right)=\left\{\begin{array}{l}
1, \\
1-\lambda\left(t_{j}-d_{j}\right)^{\beta}, \quad d_{j} \leq t_{j}<R_{j}, \\
0, \\
c_{j} \geq R_{j}
\end{array}\right. \\
& c_{i} \geq \sum_{j=1}^{m}\left(a_{i j}+b_{i j}+c_{i j}\right)\left(p_{j}+r_{j}\right), \\
& a_{i j}=D_{i j} x_{j}, \\
& b_{i j}=\frac{1}{2} D_{i j} x_{j}, \\
& \frac{1}{3} D_{i j} x_{j},
\end{aligned}
$$

$$
\begin{aligned}
& \sum_{i=1}^{n}\left(a_{i j}+b_{i j}+c_{i j}\right)=1, \\
& \sum_{j=1}^{m}\left(a_{i j}+b_{i j}+c_{i j}\right) \leq m, \\
& x_{j} \in\{0,1\}, \\
& D_{j} \in\{0,1,2,3\} .
\end{aligned}
$$

In this model, formula (6) is the main objective function, formula (7) is the secondary objective function, and the rest of formulas from formula (8) to formula (14) are constraints. Formula (8) measures the delay time compared with expected waiting time of patient $j$. Formula (9) measures patient satisfaction degree. Formula (10) ensures the rationality of the patients' expected waiting time. Formula (11) ensures each doctor's work time is limited in any one of the doctor outpatient departments. Formula (12) means the registration type which patient $j$ chooses to get service. Formula (13) ensures that each patient just only enters into one doctor outpatient departments and receives service for only once. Formula (14) ensures that the number of opened doctor outpatient departments is less than the total number of doctor outpatient departments. Formula (15) ensures the range of decision variables.

\section{Algorithm Design}

Since this problem about optimal scheduling of doctor outpatient departments belongs to the multiobjective combinatorial optimization. The number of combinations has an exponential relationship with the number of patients, so using the traversal method to search results is not feasible. In our article, the plant growth simulation algorithm (PGSA) is used to solve this problem. PGSA is the algorithm which applies the rules of plant phototropism into solving optimal scheduling problem. This algorithm compares the feasible region of optimal problem to plant growth environment and it compares global optimum solution to light which the plant requires to grow. So it can simulate the plant phototropism growth rules and then build growth model of tree trunks and branches under the action of different morphactins. In recent years, many scholars put PGSA into different research areas and have received satisfactory results. Such as Xuping et al. [19] in solving the distributive problem of emergency supplies, Qingkui et al. [20] in solving vehicle scheduling problem and Yang et al. [21] in solving scheduling of the bottleneck in auto maintenance shop are all using this algorithm.

4.1. Coding Design. Firstly, we number the patients and then encode them according to the way of real number. An array shows a treatment plan and the length of array is $m+n-1$ and we use $-1 \sim-(m+1)$ as identifiers of different clinics. The array of Figure 6 shows the case that there are ten patients and four clinics. We can know which clinic that patients enter according to above number. For example, in Table 1, the patient number 10 is in number 3 clinic. 
TABLE 1: The coding example.

\begin{tabular}{lllllllllllll}
\hline 3 & 4 & -1 & 6 & 8 & -2 & 7 & 10 & 2 & 5 & -3 & 1 & 9 \\
\hline
\end{tabular}

4.2. The Probability Model of Plant Growth Simulation Phototropism. We assume the growth process of plant including roots, trunks, and branches. The initial feasible solution $x_{0}$ of the problem represents roots and the number of initial growing point is $s$ which are in trunk $L$. These initial growing points are denoted by $A_{L 1}, A_{L 2}, \ldots, A_{L s}$ and the corresponding morphactins are, respectively, denoted by $P_{L 1}, P_{L 2}, \ldots, P_{L s}$. The number of initial growing point is $r$ which are in branch $H$. These initial growing points are denoted by $A_{H 1}, A_{H 2}, \ldots, A_{H r}$ and the corresponding morphactins are, respectively, denoted by $P_{H 1}, P_{H 2}, \ldots, P_{H r}$. The computational formulas are as follows:

$$
\begin{aligned}
& P_{L i} \\
& =\frac{f\left(x_{0}\right)-f\left(A_{L i}\right)}{\sum_{i=1}^{s}\left(f\left(x_{0}\right)-f\left(A_{L i}\right)\right)+\sum_{i=1}^{r}\left(f\left(x_{0}\right)-f\left(A_{H i}\right)\right)}, \\
& P_{H i} \\
& =\frac{f\left(x_{0}\right)-f\left(A_{H i}\right)}{\sum_{i=1}^{s}\left(f\left(x_{0}\right)-f\left(A_{L i}\right)\right)+\sum_{i=1}^{r}\left(f\left(x_{0}\right)-f\left(A_{H i}\right)\right)} .
\end{aligned}
$$

In the formulas, $f(*)=1 / Z$ represents the objective function value of growing point and the goal of this algorithm is to search growing point which minimizes the value of $f(*)$. After determining the morphactin, we can decide whether to produce new branches in existing branches according to the value of morphactin. It means that the higher morphactin concentration of growing point will receive prior opportunity. According to formula (16), we know that $\sum_{i=1}^{s} P_{L i}+\sum_{i=1}^{r} P_{H i}=$ 1. First of all, we use computer generated random number in the domain of $[0,1]$ and then use the way of throwball to select prior growing point. After generating a new growing point, we should compute its objective function value and update the value of morphactin. Repeat this process until there is no new branch and then we can obtain the optimal solution.

\subsection{Algorithm Steps}

Step 1. Generate initial feasible solution randomly according to the way of encoding we designed and then calculate the value of $f\left(x_{0}\right)$. Firstly, $x_{\min }=x_{0}, f_{\min }\left(x_{\min }\right)=f\left(x_{0}\right)$.

Step 2. Based on the root $x_{0}$, it generates 200 new growing points according to the method of two-point switching for improving the efficiency of search. The first switching position generates randomly and the next switching position generates according to the way of increasing four bits. If this position is beyond the individual interval after increasing four bits, the remaining bits should be researched from the initial part of individual again. It is similar to treating individual as a closed loop. If there are the same values in switching interval under adopting such operation, we make second adjustment of bits which have the same value and allow adjusting bits in switching interval in order to eliminate generating individual wrongly. Verify the feasibility of growing points according to constraints.

Step 3. Calculate the objective function value of growing points and then compare them with $f_{\text {min }}$. If the objective function value of growing points is less than $f_{\min }$, then update $x_{\min }, f_{\min }\left(x_{\min }\right)$.

Step 4. Calculate the morphactin value of feasible growing points according to formula (16).

Step 5. Use computer generated random number in the domain of $[0,1]$ and then use the way of throwball to select the next prior growing point.

Step 6. Repeat Step 2 Step 3 until new growing point stopped generating or reached the number of iterations.

Step 7. Choose the optimal solution which minimizes the number of doctor outpatient departments and then determine the optimal scheduling scheme.

\section{Case Study}

A survey has been carried out in a high-level hospital in Harbin, China, and the goal is to make the optimal scheduling of doctor outpatient departments for the peak attendance period (8:00-10:00). The total number of doctor outpatient departments is 10 , and the general-clinic's number is 4 , the specialist-clinic's number is 3 , and the emergency-clinic's number is 3 . The average service time of general-clinic is 10 minutes and the service rate is 6 persons per hour; the average service time of specialist-clinic is 20 minutes and the service rate is 3 persons per hour and the average service time of emergency-clinic is 8 minutes and the service rate is 7.5 persons per hour. The total number of patients under this period is 112. The arrival rate of patients is $\eta=0.02$ person/s which obey Poisson distribution. We use MATLAB to solve this scheduling problem. The number of iterations is set 89 times and it runs five times under the Windows 8 platform with RAM of $4 \mathrm{~GB}$ and basic frequency is $2.30 \mathrm{GHz}$. Its average running time is $68 \mathrm{~s}$. In the steady state, the number of general-clinic is 3; the number of specialist-clinic is 3; and the number of emergency-clinic is 1 ; and the ultimate patient satisfaction degree is 0.92 . The optimization results are shown in Table 2.

The row of "State" represents whether the doctor outpatient department is open. " 1 " indicates that it is open and " 0 " indicates that it is closed.

In order to analyze the performance of PGSA, we compare results in Table 2 with the results of traditional genetic algorithm (GA). The initialized population of GA is 200, the crossover rate is 0.85 , the mutation rate is 0.05 , and the number of iterations is set 89 times. The two algorithms run 5 times, respectively, under the same constraints and configuration of the computer. The convergence process of optimal solution is shown in Figure 7 . In the figure, $x$-axis 
TABLE 2: Optimization results.

\begin{tabular}{|c|c|c|c|c|c|c|c|c|c|c|}
\hline \multirow[b]{3}{*}{ Number } & \multicolumn{10}{|c|}{ Category } \\
\hline & \multicolumn{4}{|c|}{ General-clinic } & \multicolumn{3}{|c|}{ Specialist-clinic } & \multicolumn{3}{|c|}{ Emergency-clinic } \\
\hline & 1 & 2 & 3 & 4 & 1 & 2 & 3 & 1 & 2 & 3 \\
\hline State & 1 & 1 & 1 & 0 & 1 & 1 & 1 & 1 & 0 & 0 \\
\hline The number of patients & 21 & 21 & 20 & 0 & 12 & 12 & 13 & 13 & 0 & 0 \\
\hline
\end{tabular}

TABLE 3: The comparative analysis results of PGSA and GA.

\begin{tabular}{lccccc}
\hline Algorithm & $\begin{array}{c}\text { The number of } \\
\text { general-clinics }\end{array}$ & $\begin{array}{c}\text { The number of } \\
\text { specialist-clinics }\end{array}$ & $\begin{array}{c}\text { The number of } \\
\text { emergency-clinics }\end{array}$ & $\begin{array}{c}\text { Satisfaction degree } \\
\text { CPU }\end{array}$ \\
\hline GA & 3 & 2 & 2 & 0.88 & 75 \\
PGSA & 3 & 3 & 1 & 0.92 & 68 \\
\hline
\end{tabular}

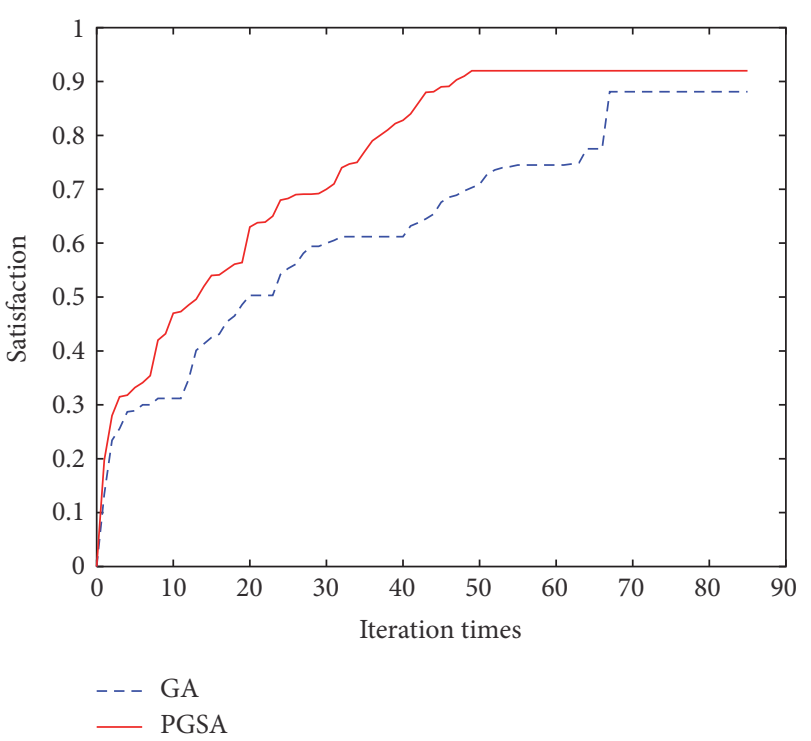

FIGURE 7: The optimal solution of convergence.

indicates the number of algorithm iterative times; $y$-axis indicates the average satisfaction degree of patients and we can see that patients' average satisfaction degree is increasing. Compared with GA, PGSA is able to stabilize the value in less iterations and it has characteristics of high convergence speed and efficiency which can meet patients' psychological needs minimizing the waiting time better. The comparative optimal result of PGSA and GA is shown in Table 3.

The results' simulation shows that setting appropriate number of doctor outpatient departments under different types of clinics can improve patient satisfaction effectively and reduce hospital operating costs at the same time and then achieve excellent management. Thus, adjusting the doctor outpatient departments' quantity under different types of clinics according to dynamic periods is very necessary choice in hospital.

\section{Conclusion}

Patients are main part of hospital, so it is very significant to improve patients' satisfaction. In this paper, an optimal scheduling strategy has been designed for the problem of patients queuing in the hospital. Firstly, the patient behavioral characteristics are analyzed, and then the patient membership functions and basic model are built to make optimal scheduling for doctor outpatient departments. Secondly, PGSA is used to solve this problem. Comparing with traditional algorithm GA, it takes less time to find the optimal solution which has better stability. The PGSA can solve optimal scheduling of doctor outpatient departments effectively in order to increase patient satisfaction and at the same time reduce operating costs in hospital. Finally, the simulation results of an example have proved the correctness and validity of the model and PGSA we designed. The paper focuses on different types of registrations without considering the different types of doctor outpatient departments. In the future, different types of doctor outpatient departments as a variable will be added in order to make the research of this problem close to reality greatly.

\section{Competing Interests}

The authors declare that they have no competing interests.

\section{Acknowledgments}

This work is supported by the National Nature Science Foundation of China (71371061) and Postdoctoral Foundation of Heilongjiang Province (LBH-Z15108).

\section{References}

[1] S. Mahar, K. M. Bretthauer, and P. A. Salzarulo, "Locating specialized service capacity in a multi-hospital network," European Journal of Operational Research, vol. 212, no. 3, pp. 596-605, 2011. 
[2] N. Meskens, D. Duvivier, and A. Hanset, "Multi-objective operating room scheduling considering desiderata of the surgical team," Decision Support Systems, vol. 55, no. 2, pp. 650-659, 2013.

[3] J. R. Villamizar, F. C. Coelli, W. C. A. Pereira, and R. M. V. R. Almeida, "Discrete-event computer simulation methods in the optimisation of a physiotherapy clinic," Physiotherapy, vol. 97, no. 1, pp. 71-77, 2011.

[4] P. Landa, R. Aringhieri, P. Soriano, E. Tànfani, and A. Testi, "A hybrid optimization algorithm for surgeries scheduling," Operations Research for Health Care, vol. 8, pp. 103-114, 2016.

[5] J. L. Vile, J. W. Gillard, P. R. Harper, and V. A. Knight, "Timedependent stochastic methods for managing and scheduling Emergency Medical Services," Operations Research for Health Care, vol. 8, pp. 42-52, 2016.

[6] P. D. Wright and S. Mahar, "Centralized nurse scheduling to simultaneously improve schedule cost and nurse satisfaction," Omega, vol. 41, no. 6, pp. 1042-1052, 2013.

[7] D. A. Stanford, J. M. Lee, N. Chandok, and V. McAlister, "A queuing model to address waiting time inconsistency in solidorgan transplantation," Operations Research for Health Care, vol. 3, no. 1, pp. 40-45, 2014.

[8] S. P. J. van Brummelen, W. L. de Kort, and N. M. van Dijk, "Waiting time computation for blood collection sites," Operations Research for Health Care, vol. 7, pp. 70-80, 2015.

[9] J. T. van Essen, E. W. Hans, J. L. Hurink, and A. Oversberg, "Minimizing the waiting time for emergencysurgery," Operations Research for Health Care, vol. 1, no. 2-3, pp. 34-44, 2012.

[10] L. Shuangxi, S. Yi, and J. Lian, "Establishing an outpatient service management system that suits the time pattern of patients' visits," Chinese Journal of Hospital Administration, vol. 17, no. 5, pp. 296-298, 2001.

[11] C. Lakshmi and S. A. Iyer, "Application of queueing theory in health care: a literature review," Operations Research for Health Care, vol. 2, no. 1-2, pp. 25-39, 2013.

[12] W. Xuping, M. Chao, and R. Junhu, "Emergency supplies optimal scheduling considering the public's psychological risk perception," System Engineering Theory and Practice, vol. 33, no. 7, pp. 1735-1742, 2013.

[13] J. Yang, S. Wei, and D. Qiulei, "Model of disruption management with actors in single machine scheduling," Journal of Mechanical Engineering, vol. 49, no. 14, pp. 191-198, 2013.

[14] Z. Zhihong, K. Jisong, and C. Fuzan, "Feature extraction of customer purchase behavior based on genetic algorithm," Pattern Recognition and Artificial Intelligence, vol. 23, no. 2, pp. 256-266, 2010.

[15] J. Chun and Z. Yiping, "Agent-based simulation model of customer behavior and personalized recommendation," Systems Engineering-Theory \& Practice, vol. 33, no. 2, pp. 463-472, 2013.

[16] W. Tingting, Y. Qin, and H. Lin, "Optimization scheduling of retail checkout based on customers' behavior," Computer Engineering and Applications, vol. 52, no. 3, pp. 266-270, 2016.

[17] D. Kahneman and A. Tversky, "Prospect theory: an analysis of decision under risk," Econometrica, vol. 47, no. 2, pp. 263-291, 1979.

[18] Z. Jianmin, "An experimental test on cumulative prospect theory," Journal of Jinan University (Natural Science), vol. 28, no. 1, pp. 44-48, 2007.

[19] W. Xuping, Z. Nana, and Z. Hongxin, "Emergency material allocation model considering the non-rational psychological comparison of the victims," Chinese Journal of Management, vol. 13, no. 7, pp. 1075-1080, 2016.
[20] C. Qingkui, L. Xinyu, and R. Xiangyang, "Vehicle scheduling problem based on plant growth simulation algorithm," Systems Engineering-Theory Practice, vol. 35, no. 6, pp. 1449-1456, 2015.

[21] Q. Yang, G.-H. Zhou, and J.-J. Lin, "Scheduling of machines in parallel with different speeds based on plant growth simulation algorithm-taking an example of the bottleneck in $4 \mathrm{~S}$ auto dealership maintenance shop," System Engineering Theory and Practice, vol. 32, no. 11, pp. 2433-2438, 2012. 

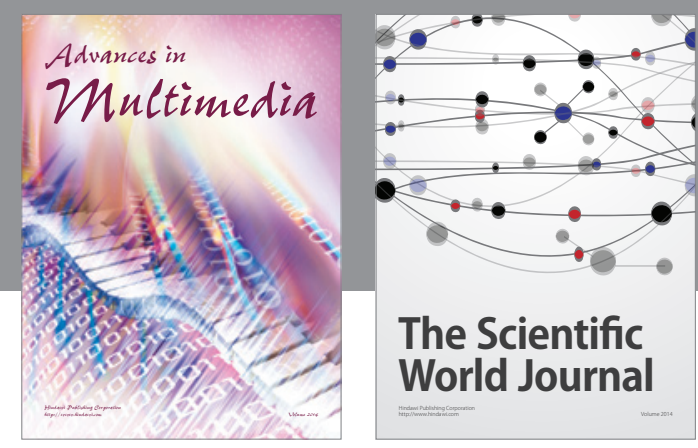

The Scientific World Journal
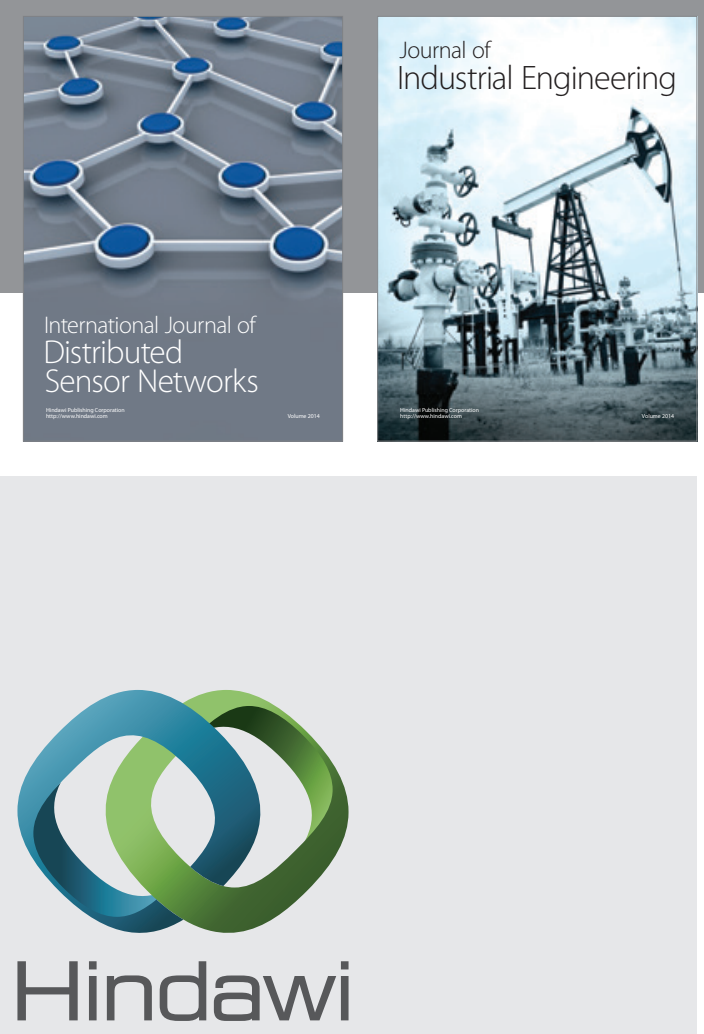

Submit your manuscripts at

http://www.hindawi.com

\section{Computer Networks} and Communications
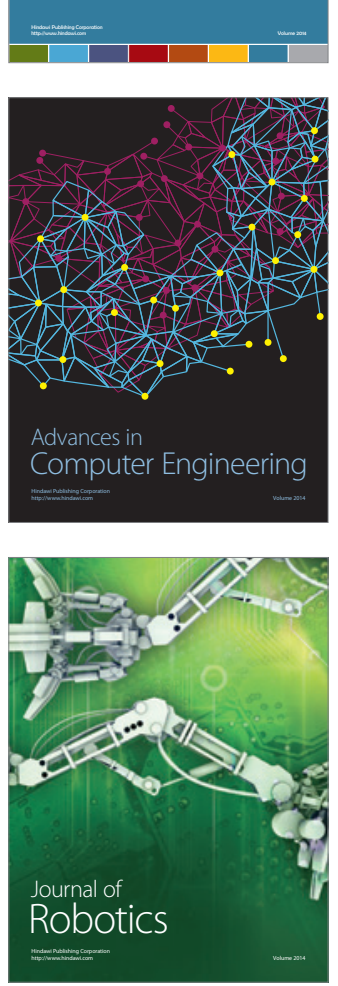
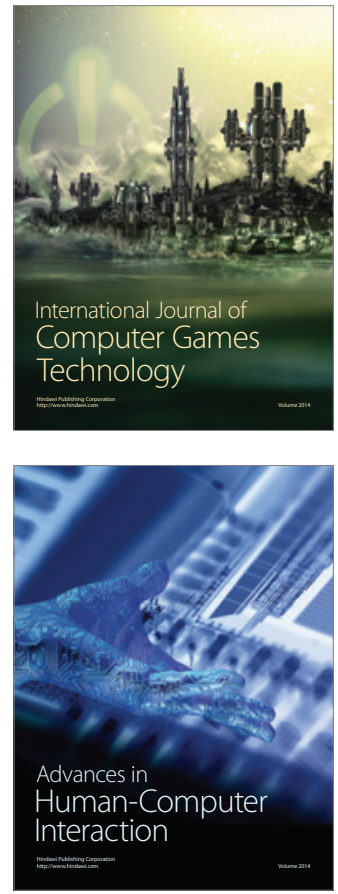
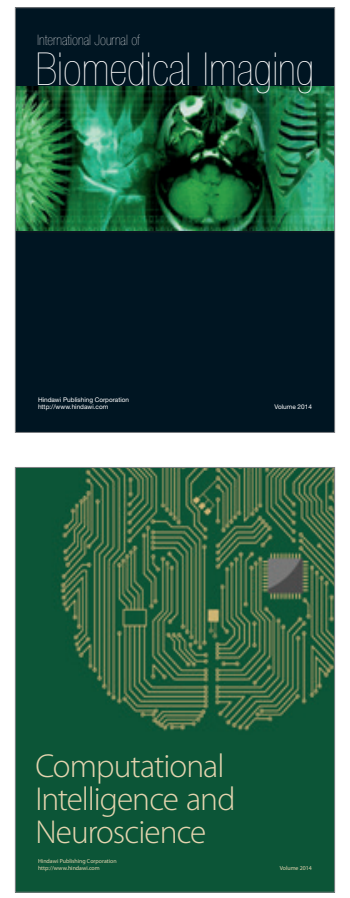
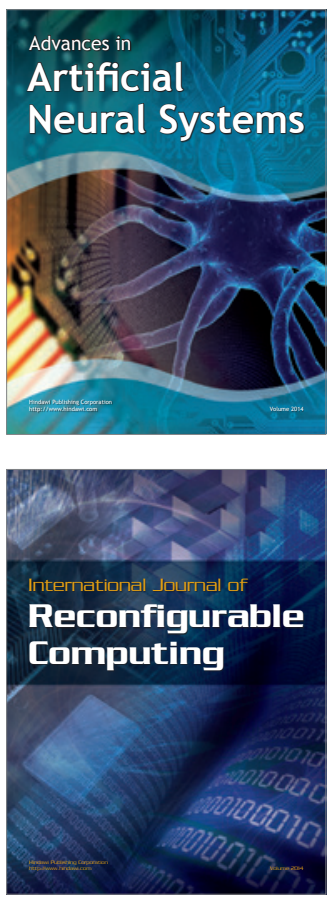
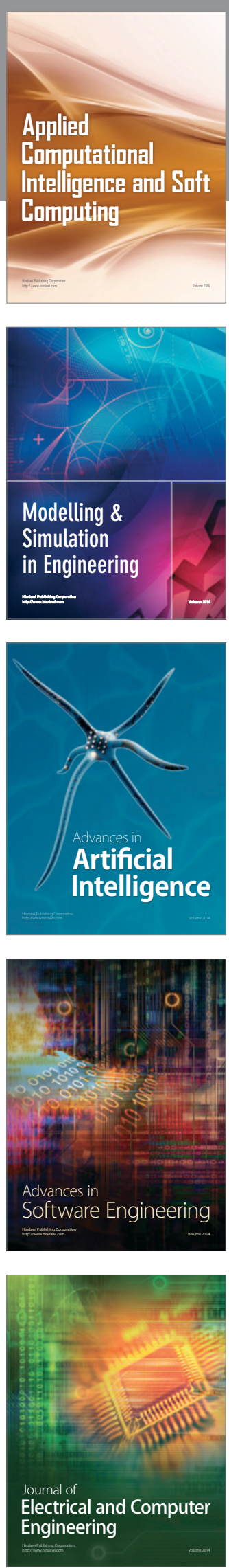